OPEN ACCESS

Edited by: Ivan Mijakovic, Chalmers University of Technology, Sweden

Reviewed by:

Ralf J. Braun,

Universität Bayreuth, Germany

Lim Boon Huat,

Universiti Sains Malaysia, Malaysia

*Correspondence:

Qun Liu

qunliu@cau.edu.cn

Specialty section:

This article was submitted to Microbial Physiology and Metabolism,

a section of the journal

Frontiers in Microbiology

Received: 12 October 2015 Accepted: 23 December 2015

Published: 19 January 2016

Citation:

Li M, Wang H, Liu J, Hao P, Ma L and Liu Q (2016) The Apoptotic Role of

Metacaspase in Toxoplasma gondii.

Front. Microbiol. 6:1560

doi: $10.3389 /$ fmicb.2015.01560

\section{The Apoptotic Role of Metacaspase in Toxoplasma gondii}

\author{
Muzi Li, Hui Wang, Jing Liu, Pan Hao, Lei Ma and Qun Liu *
}

Key Laboratory of Animal Epidemiology and Zoonosis, Ministry of Agriculture, National Animal Protozoa Laboratory, College of Veterinary Medicine, China Agricultural University, Beijing, China

Toxoplasma gondii is a major opportunistic pathogen that spreads in a range of animal species and human beings. Quite a few characterizations of apoptosis have been identified in T. gondii treated with apoptosis inducers, but the molecular mechanisms of the pathway are not clearly understood. Metacaspases are caspase-like cysteine proteases that can be found in plants, fungi, and protozoa in which caspases are absent. Metacaspases are multifunctional proteases involved in apoptosis-like cell death, insoluble protein aggregate clearance, and cell proliferation. To investigate whether $T$. gondii metacaspase (TgMCA) is involved in the apoptosis of the parasites, we generated TgMCA mutant strains. Western blot analysis indicated that the autoproteolytic processing of TgMCA was the same as that for metacaspases of some other species. Indirect immunofluorescence assay (IFA) showed that TgMCA was dispersed throughout the cytoplasm and relocated to the nucleus when the parasites were exposed to the extracellular environment, which indicated the execution of its function in the nucleus. The number of apoptosis parasites was significantly diminished in the TgMCA knockout strain and increased in the TgMCA overexpression strain after treatment with extracellular buffer, as determined by the terminal deoxynucleotidyl transferase dUTP nick end labeling (TUNEL) assay. The lack of TgMCA did not affect the parasite propagation in vitro and virulence in vivo, suggesting that it is probably redundant in parasite propagation. But overexpression of TgMCA reduced the intracellular parasites growth in vitro. The TgMCA knockout strain showed more viability in extracellular buffer compared to the parental and overexpression lines. In this study, we demonstrated that TgMCA contributes to the apoptosis of $T$. gondii.

Keywords: T. gondii, apoptosis, programmed cell death, metacaspase, knockout

\section{INTRODUCTION}

Apoptosis, which is a form of programmed cell death (PCD), is important to the development and homeostasis of metazoans (Elomre, 2007). However, it remains controversial in protozoa because it leads to the death of a single-celled organism; how apoptosis might benefit a unicellular eukaryote is also difficult to understand (Reece et al., 2011). Recently, increasing evidence has demonstrated that regulated cell death occurs in single-celled organisms, although the dedicated molecular mechanism, and function remain unclear (Kaczanowski et al., 2011). To date, apoptosis has been described in Trypanosoma, Leishmania, Giardia lamblia, and Plasmodium falciparum, among other species (Bruchhaus et al., 2007; Gannavaram and Debranbant, 2012; Proto et al., 2013). Moreover, hallmarks of apoptosis in metazoans, including shrinkage and rounding of 
the body shape, externalization of phosphatidylserine (PS), DNA fragmentation and nucleus condensation, were observed and identified in the protozoa mentioned above, either naturally or under stimulation (Jiménez-Ruiz et al., 2010; Proto et al., 2013). Van Zandbergen et al. reported that Leishmania promastigotes contain a high ratio of apoptotic parasites that enable the intracellular survival of viable parasites in an altruistic way (van Zandbergen et al., 2006; Wanderley et al., 2013). The stumpy trypomastigote of Trypanosoma brucei, if it is not taken up by a tsetse fly for transmission, will undergo apoptosis in the mammalian host (Laverrière et al., 2012). Toxoplasma gondii appears to exhibit apoptotic characteristics when treated with $\mathrm{H}_{2} \mathrm{O}_{2}$, and the externalization of phosphatidylserine of parasites was identified in the peritoneal lavage fluids of mice infected with T. gondii by intraperitoneal injection (Santos et al., 2011; Nyoman and Lüder, 2013).

The mechanism of apoptosis in protozoa has not been studied in depth. Most of the genes that have been identified as participating in apoptotic pathways in metazoans are absent in the genome of parasitic protozoa. Hence, we hypothesized that the apoptosis pathway of protozoa may differ from that of mammals. Caspases are the central cysteine peptidases involved in apoptosis, but it is thought that protozoa might lack caspases. Metacaspases, orthologous to caspases, are found in plants, fungi, and protozoa in which caspases are absent (Uren et al., 2000). Metacaspases also belong to clan CD, C14 family, which exhibit significant structural similarity and a catalytic cysteine-histidine dyad compared with caspases (Carmona-Gutierrez et al., 2010). It is widely believed that the two types of metacaspases can be distinguished by the $\mathrm{N}$-terminal prodomain, though another new one type of metacaspase has been found in phytoplankton (Choi and Berges, 2013). Type I metacaspases have an Nterminal prodomain, whereas type II metacaspases lack this prodomain but harbor a linkage between the p20 and p10 domains (Tsiatsiani et al., 2011). In protozoa and fungi, only type I metacaspases are found. More and more reports of functions of metacaspase are being published, and apoptosis has become a research focus. A cell death role for yeast Ycal (the only metacaspase in Saccharomyces cerevisiae) was first demonstrated in budding yeast cell death (Madeo et al., 2002). Kosec et al. showed that TcMCA5 could be involved in the apoptosis of the parasites, as it was found to relocate from the cytoplasm to the nucleus during apoptosis induced by fresh human serum (Kosec et al., 2006). In Leishmania species, metacaspases were required for oxidative stress-induced cell death (Zalila et al., 2011). In the plant Norway spruce, metacaspase (mcII-Pa) causes developmental arrest at the early stage of embryogenesis by PCD (Suarez et al., 2004).

T. gondii is a major opportunistic pathogen that produces asymptomatic infection and cyst formation in the brains of normal people but can cause disease in immunosuppressed patients (Dubey, 2010). We conducted a comprehensive search in the Toxoplasma database (ToxoDB), and found three genes encoding metacaspase-like proteins in T. gondii. Here, we report the function of one of metacaspase in apoptosis and the phenotypic changes by the analysis of metacaspase null mutants.

\section{MATERIALS AND METHODS}

\section{Animal Approval Statements}

Our research with all animals were approved by the Beijing Laboratory Administration Committee in accordance with the recommendations in the Guide for the Care and Use of Laboratory Animals of the Ministry of Science and Technology of China (Approval No.: 18049).

\section{Parasites and Cell Cultures}

Human foreskin fibroblast (HFF) and African Green Monkey kidney cell (Vero) lines were cultured in Dulbecco's Modified Eagle's Medium (DMEM) containing $25 \mathrm{mM}$ glucose and $4 \mathrm{mM}$ glutamine supplement with 10\% fetal bovine serum (FBS, Gibco, USA), as previously described (Wang et al., 2014). T. gondii RH $\Delta$ ku80 (The Ku80 gene of the parasites had been deleted to improve the efficiency of gene targeting via double-crossover homologous recombination) tachyzoites (trophozoites, a stage of T. gondii, can infect almost any cell in the body and divides by endodyogeny) were maintained in vitro by serial passages on confluent Vero cells. Both cells and parasites were incubated at $37^{\circ} \mathrm{C}$ with $5 \% \mathrm{CO}_{2}$ in a humidified incubator.

\section{Bioinformatics Analysis of TgMCA}

The gene sequence and amino acid sequence of $\mathrm{TgMCA}$ were obtained from ToxoDB (http://www.toxoDB.org, ver9.0). For further understanding, motif analysis (http://www.expasy. org/prosite), SMART (http://smart.embl-heidelberg.de/), and conserved domain predicition (CD research, http://www.ncbi. nlm.nih.gov) were used to obtain protein motifs and domains. The similarities to related proteins were analyzed by DNAman (Lynnon Biosoft, USA) and Mega5.0 (http://www.megasoftware. net). Sequence alignment analysis and three-dimensional structure modeling were performed using Espript3.0 (http:// espript.ibcp.fr/ESPript/cgi-bin/ESPript.cgi) and Swiss model (http://www.swissmodel.expasy.org/).

\section{Cloning of TgMCA and Preparation of Anti-rTgMCA Polyclonal Antibody}

Based on the gene sequence of TgMCA, a PCR process was designed, but the complete coding sequence could not be obtained directly. Therefore, overlapping primers (Table S2) were designed to amplify the complete coding sequence, partly from $\mathrm{RH} \Delta \mathrm{ku} 80 \mathrm{cDNA}$ prepared from total RNA and partly from the RH $\Delta \mathrm{ku} 80$ genome prepared using a DNA extraction kit (Aidlab Biotechnologies Co., Ltd., Beijing). Overlapping PCR was designed to obtain the complete coding sequence of TgMCA using high-fidelity polymerase (Fastpfu, TansGenBiotech Co., Ltd., Beijing). The fragment was cloned into the pEASY-T blunt vector (TansGenBiotech Co., Ltd., Beijing), and sequencing result was correct. Then the complete coding sequence was cloned into the pET-28a vector (Novagen, Germany) and transformed into Escherichia coli (E. coli, Transetta, TansGenBiotech Co., Ltd., Beijing) for expression. The rTgMCA was purified by affinity chromatography using Ni-IDA agarose (Novagen, Germany) following the Laboratory Manual of Molecular 
Cloning (Sambrook and Russell, 2000). The purified protein was assessed by SDS-PAGE.

Anti-TgMCA antibodies were obtained from 8 week-old female Balb/c mouse by immunizing $100 \mu \mathrm{g}$ recombinant protein with the same volume of Freund's Adjuvant (Sigma, USA) per mouse. Anti-r TgMCA serum was collected 2 weeks after the last immunization. The titers of polyclonal antibodies were examined by enzyme-linked immunosorbent assay (ELISA) using rTgMCA as antigen. All serum samples were sterilized by filtration through $0.22 \mu \mathrm{m}$ filters (Millipore, USA) and cryopreserved at $-80^{\circ} \mathrm{C}$.

\section{Western Blot}

Freshly isolated parasites were harvested and purified by filtration through a $5 \mu \mathrm{m}$ filter, collected by centrifugation at $1400 \times \mathrm{g}$ for $10 \mathrm{~min}$ and washed in phosphate-buffer saline (PBS). Purified parasites were lysed with RIPA buffer (50 mM Tris pH7.4, $150 \mathrm{mM} \mathrm{NaCl}, 1 \%$ Triton X-100, $1 \%$ sodium deoxycholate, $0.1 \%$ SDS, Beyotime, Shanghai) with the protease inhibitor PMSF (Beyotime, Shanghai), and 7-10 $\mu \mathrm{g}$ of lysate was used for SDS-PAGE (12\% w/v) electrophoresis and transferred onto polyvinylidene fluoride (PVDF) membranes (Millipore, USA). The membranes were blocked with 5\% (w/v) skim milk in PBS. Then, membranes were incubated for $1 \mathrm{~h}$ at room temperature with mouse anti-r $T g \mathrm{MCA}$ antibody or mouse anti-HA monoclone antibody (Santa Cruz Biotechnology, Inc., USA), diluted 1:500 in 5\% skim milk in PBS and goat anti-mouse $\operatorname{IgG}(\mathrm{H}+\mathrm{L})$ horseradish peroxidase (Sigma, USA) as secondary antibody, diluted 1:5000 in 5\% skim milk in PBS, and incubated at room temperature. Finally, chemiluminescence reagents (CoWin Biotech Co., Ltd., Beijing) were used for reactive band visualization.

\section{Immunofluorescence Assay}

Immunofluorescence assays (IFA) for TgMCA subcellular localization were performed as described previously (Wang et al., 2014). Appropriate amounts of parasites were seeded onto HFFs previously prepared on glass coverslips in 12 -well plates. Infected cells were incubated at $37^{\circ} \mathrm{C}$ with $5 \% \mathrm{CO}_{2}$ for no more than $20 \mathrm{~h}$ and fixed for $15 \mathrm{~min}$ in $4 \%$ formaldehyde, and then permeabilized with $0.25 \%$ Triton X-100 for $15 \mathrm{~min}$ and blocked with $3 \%$ bovine serum albumin (BSA) for $30 \mathrm{~min}$. Subsequently, incubation was performed with mouse anti-rTgMCA polyclonal antibody diluted $1: 50$ at $37^{\circ} \mathrm{C}$ for $1 \mathrm{~h}$, then incubation was performed with FITC-conjugated goat-anti mouse IgG(H+L) (Sigma, USA) diluted $1: 100$ with $3 \% \mathrm{BSA}$ for $1 \mathrm{~h}$ at $37^{\circ} \mathrm{C}$. DNA of nucleus was stained with Hoechst33258 (Sigma, USA) for $5 \mathrm{~min}$. The coverslips were observed and images were obtained using a Leica confocal microscope system (Leica, TCS SP52, Germany) with an oil immersion lens with $63 \times$ magnification and the maximum emission wavelength of the fluorescent antibody $(525 \mathrm{~nm}$ for FITC and $570 \mathrm{~nm}$ for cy3). Rabbit anti-T. gondii polyclonal antibody were used to stain the shape of the tachyzoites and pre-immune mouse serum were used as controls. The brightness and contrast of the images were adjusted using the LAS AF lite 2.2.0 software and the images were exported from this software. To analyze $T g M C A$ localization in extracellular parasites, filtered parasites were allowed to adhere to coverslips precoated with poly-lysine at $4^{\circ} \mathrm{C}$ overnight, and the IFA process and images collection were performed as above.

\section{Generation of TgMCA Knockout and Overexpression Strains}

The parental strain used to generate the knockout strain was RH $\Delta$ ku80, which was transfected with the complete knockout plasmid PTCR-CD including genes of chloramphenicol and RFP (T. gondii positive selected marker gene) and the $\mathrm{CD}$ gene (bacterial cytosine deaminase, $T$. gondii negative selected marker gene). Briefly, $\sim 2000 \mathrm{bp}$ of the $5^{\prime}$ flanking and $3^{\prime}$ flanking sequence of TgMCA were amplified from the RH $\Delta$ ku 80 genome (primers in Table S3). The products were flanked with XhoI and HindIII, XmaI, and XbaI, respectively, and cloned into the PTCR-CD vector in order. The knockout plasmid was named PTCR-CD TgMCA KO. PTCR-CD TgMCA KO was used to disrupt the native loci of $\Delta \mathrm{ku} 80$ tachyzoites by the double homologous recombination and replacement of the entire coding region, and stable clones were derived by chloramphenicol selection. Primers were designed to identify the correct clones (Figure 3A, Table S4), and finally the clones were confirmed by Western blot. For complementation analysis, the CRISPR/CAS9UPRT specific sgRNA (kindly provided by Prof. BangShen, Shen et al., 2014) was used for the targeted disruption of the uracil phosphoribosyl transferase (UPRT) gene and used with resistance to fluorodeoxyribose (FUDR) for negative selection. Meanwhile, the TgMCA complete coding sequences under the $T g$ GRA 1 promoter flanked by UPRT $5^{\prime}$ and $3^{\prime}$ flanking sequences were co-transfected into $T g \mathrm{MCA}$ knockout strain $(\triangle \mathrm{TgMCA})$ and the parasites were selected by FUDR. To further characterize the role of $T g \mathrm{MCA}$, we generated a transgenic RH $\Delta \mathrm{ku} 80$ strain overexpressing $\mathrm{TgMCA}$. The complete coding sequence of $T g$ MCA was amplified using primers that appended the flanking EcoRV and AvrII restriction endonuclease sites. Amplification products were introduced into the same site of a modified pDMG plasmid which GFP was replaced by HA, and the vector pDMG-TgMCA-HA was electroporated into $\Delta$ ku 80 for $T g \mathrm{MCA}$ overexpression. The transgenic parasites were grown under selection pressure of pyrimethamine. The clones were identified by Western blot as described above.

\section{Plaque Assay}

The plaque assay was performed on HFF cells cultured in 6well plates (Corning costar, USA) as described previously (Wang et al., 2014). A total of 500 freshly isolated parasites were seeded into HFF monolayers, and incubated at $37^{\circ} \mathrm{C}$ with $5 \% \mathrm{CO}_{2}$ for 7 days undisturbed. After 7 days, the medium was removed from the HFFs, and they were washed 3-5 times with PBS. The cell monolayers were fixed with $4 \%$ formaldehyde for $10 \mathrm{~min}$, stained with $0.2 \%$ crystal violet solution for $30 \mathrm{~min}$ and washed with deionized water, then visualized by microscopy (Olympus Co., Japan). The 6-well plates were scanned using an Canon digital scanner (Model: F917500, Japan). At least 50 plaques of each strain were chosen randomly and the plaque area was counted using Pixel in the Photoshop C6S software(Adobe, USA), and the data were compiled from three independent 
experiments. Statistical analysis was performed using One-way ANOVA with Tukey's post-hoc comparison. Differences were considered significant if the $P \leq 0.05$.

\section{Intracellular Parasite Replication Assay}

Freshly isolated parasites $\left(1 \times 10^{6}\right)$ were inoculated on HFFs in 12-wellplates (Corning costar, USA). After $30 \mathrm{~min}$, the extracellular parasites were removed by washing 3-5 times with PBS and the plates were checked by microscope for the uninvaded parasites were removed completely. After incubation for $20 \mathrm{~h}$, the infected cells were fixed with $4 \%$ formaldehyde, and the parasites were stained using rabbit anti- $T$. gondii positive serum following the IFA protocol. The number of parasites per vacuole were counted for each strain using a fluorescence microscope (Olympus Co., Japan) at $400 \times$ magnification, and a total count of 100 parasitophorous vacuoles (PVs) of each strain was performed in each of three independent experiments. Statistical analysis was performed with the Chi-square test using statistical analysis system (SAS institute Inc., USA). Differences were considered significant if the $P \leq 0.05$.

\section{Invasion Assay}

For the experimental testing of invasion, freshly isolated parasites were harvested, and $1 \times 10^{6}$ parasites were seeded on HFFs in 12-well plates. After $30 \mathrm{~min}$, the extracellular parasites were removed by washing for 3-5 times with PBS and the plates were checked by microscope for the uninvaded parasites were removed completely, then incubated for $24 \mathrm{~h}$ at $37^{\circ} \mathrm{C}$ with $5 \% \mathrm{CO}_{2}$. To test the viability of extracellular parasites, parasites were pre-incubated in extracellular buffer (Ringer buffer, $155 \mathrm{mM} \mathrm{NaCl}, 3 \mathrm{mM} \mathrm{KCl}, 2 \mathrm{mM} \mathrm{CaCl}, 1 \mathrm{mM} \mathrm{MgCl}$, $3 \mathrm{mM} \mathrm{NaH}{ }_{2} \mathrm{PO}_{4}, 10 \mathrm{mM}$ HEPES, and $10 \mathrm{mM}$ glucose) for 1$2 \mathrm{~h}$ and seeded on HFF monolayers, and the extracellular parasites were removed and incubated as mentioned above. Subsequently, the medium was removed and fixed with $4 \%$ formaldehyde. To observe the parasite invasion and analyze the invasion ratio, IFA was performed following the method described above. Counting was performed using the fluorescence microscope, and data were compiled from three independent experiments, each in triplicate, counting eight randomly selected fields per clone at $400 \times$ magnification. The ratio was calculated based on the number of infected cells divided by the number of total cells in one field of view. Statistical analysis was performed using Two-way ANOVA with Tukey's multiple comparison test. Differences were considered significant if the $P \leq 0.05$.

\section{Virulence Assay in Mice}

Virulence of the parasites were performed as described previously (Wang et al., 2014). Female, 8-week-old Balb/c mice were purchased from the Laboratory Animal Center of the Academy of Military. Rodent laboratory chow and tap water were provided, maintained under specific pathogen-free conditions and acclimatized for 7 days before each experiment. Parasites were injected intraperitoneally into the mice at doses of 10 or 100 tachyzoites (5 mice for each parasite strain), and mice were monitored until there were no survivors. All the infected mice were monitored every $8 \mathrm{~h}$ for clinical signs and mortality. The mice were humanely euthanized by the subcutaneous injection of atropine $(0.02 \mathrm{mg} / \mathrm{kg})$ or by cervical dislocation when they were unable to reach food or water for more than $24 \mathrm{~h}$ and lost $20 \%$ of normal body weight. The survival data were compiled from three independent experiment (dose of 100 tachyzoite) or two independent experiment (dose of 10 tachyzoites), 5 mice per stain in each experiment. Statistical analysis was performed using life test (life test data $=$ surv) in statistical analysis system (SAS institute Inc., USA). Difference was considered significant if $p \leq 0.05$.

\section{TUNEL Assay}

The terminal deoxynucleotidyl transferase-mediated dUPT nick end labeling (TUNEL) assay was performed as recommended by the manufacturer (Vazyme Biotech, Co., Ltd, Nanjing). Freshly isolated T. gondii was harvested by centrifugation at $1400 \times$ $\mathrm{g}$ for $10 \mathrm{~min}$, washed twice with PBS, and treated with Ringer buffer as a pro-stimulus for 1-6h. Subsequently, the parasites were adhered to coverslips precoated with poly-lysine at $4^{\circ} \mathrm{C}$ overnight and fixed with $4 \%$ (w/v) paraformaldehyde in PBS for $15 \mathrm{~min}$ at room temperature. After the coverslips were washed, the parasites were permeabilized for $15 \mathrm{~min}$ with $0.1 \%$ Triton in PBS at room temperature. Then, the parasites were incubated in TUNEL reaction mix with TdT enzyme for $1 \mathrm{~h}$ at $37^{\circ} \mathrm{C}$ away from light. DNA of nucleus were stained with Hoechst33258 (Sigma, USA). To confirm the TUNEL-positive parasites, rabbit anti-T. gondii polyclonal antibody was used to stain the shape of parasites following the IFA protocol mentioned above and the samples which incubated with TUNEL reaction mix that did not contain TdT enzyme were used as control. The coverslips were observed, and images were obtained using a Leica confocal microscope system (Leica, TCS SP52, Germany). The number of TUNEL-positive parasites per image were counted at $630 \times$ magnification, and 10 images of each strain were counted in each of three independent experiments. Statistically significant analysis was performed by One-way or Two-way ANOVA with Tukey's multiple comparison test. Differences were considered significant if the $P \leq 0.05$.

\section{Statistical Analysis}

The statistical test of virulence assay and intracellular parasites replication assay were performed using statistical analysis system (SAS institute Inc., USA), and the other statistical test were performed in Statistical Product and Service Solution 22.0 (SPSS 22.0, IBM Co., USA). Data set were assumed to be equality of variance (determining by Homogeneity of variance in SPSS 22.0) and were analyzed with One-way analysis of ANOVA if only a single independent variable (e.g., genotype) or Two-way analysis of ANOVA if two independent variables (e.g., genotype and time). Tukey's post-hoc analysis was performed to comparing selected pairs of means. Two-way ANOVA was used to analyze parasites replication data, and using Chi-square for analysis each two parasite lines. In all cases, two-tailed $P$-value was calculated, and $P \leq 0.05$ was consider significant. 


\section{RESULTS}

\section{TgMCA is a Conserved Cysteine-Histidine Catalytic Dyad Protein in T. gondii}

To obtain information on metacaspases in T. gondii, we used the Toxoplasma genomic resource database (ToxoDB, ver.9.0) to search for metacaspase-related genes. Proteins containing p20 domain were found in three genotypes in $T$. gondii, all of which belong to the ICE family protein based on the annotations (TGGT1_206490, TGGT1_278975, and TGGT1_243298). The three metacaspase genes are on different chromosomes of $T$. gondii and encode proteins sharing low amino acid identity because of the long and different $\mathrm{N}$-terminal region (Table 1; Table S1). However, the three metacaspase proteins are definitely conserved in types I, II, and III T. gondii (different strains of $T$. gondii, based on the virulence to mice). We used the online service PROSITE for motif analysis and used SMART and $\mathrm{CD}$ research for conserved domain and protein structure prediction. Significant homology with the peptidase-C14/caspase domain was shown in the three metacaspases. A C2 domain (a $\mathrm{Ca}^{2+}$ dependent membrane targeting module), CUE domain (ubiquitin-binding CUE protein), and proline-rich motif were only present in TGGT1_206490, which were predicted to mediate protein-protein interaction (Figure 1D). Multiple alignment of the amino acid about the three metacaspases with Ycal (the only metacaspase in S. cerevisiae, GI: 398365705), human caspase3 (GI: 704360691), and human caspase9 (GI: 17943347) was performed by ClustalW, and interestingly, only TGGT1_206490 carries histidine and cysteine as in yeast Yca1, which are predicted to form part of the catalytic dyad specific to clan CD. The conserved histidine or histidine adjacent to cysteine was absent from the other two metacaspases (Figure 1A, indicated by red asterisk). As previously reported, the mutation of cysteine and histidine in yeast Ycal resulted in the complete abrogation of the catalytic process, and mutating the histidine adjacent to cysteine reduced the level of catalytic activity markedly (Wong et al., 2012). The ternary structure of full-length TgMCA (Figure 1B) was modeled using the structural template of yeast Yca1 according to Swiss-Model (PDB: 4f6p.1.A, Figure 1C). TgMCA encodes 742 amino acid, and 250 amino acid at Cterminal formed the $\mathrm{p} 20$ domain, which forming a middle $\beta$-sheet sandwiched by $\alpha$-helices. The conserved cysteine and histidine residues were in the loops between $\beta$-sheet 3 and $\beta$-sheet 4 and between $\beta$-sheet 7 and $\beta$-sheet 8 . Thus, in this study, we investigated the role of TGGT1_206490 (abbreviated to TgMCA) in the apoptosis of Toxoplasma by constructing TgMCA-deficient parasites.

TABLE 1 | Information on three different ICE family members containing p20 domain in ToxoDB.

\begin{tabular}{lcc}
\hline Gene ID & Chromosome & Protein length \\
\hline TGGT1_206490 & VIla & 742 \\
TGGT1_278975 & XII & 1344 \\
TGGT1_243298 & Vl & 2055
\end{tabular}

\section{TgMCA is Expressed in Tachyzoites}

To assess the expression and the localization of TgMCA in the tachyzoites, the full-length TgMCA protein fused with a histidine-tag was successfully expressed in E. coli and used to generate anti-rTgMCA serum in mice. The anti-rTgMCA polyclonal antibody reacted with an $80 \mathrm{kDa}$ protein in the lysate of tachyzoites as an antigen by western blot, and the pre-immune mouse serum exhibited no reactivity with the parasites (Figure 2A). More interestingly, two other molecular weights of positive protein bands, $\sim 65 \mathrm{kDa}$ and $15 \mathrm{kDa}$, were observed. Previous studies on metacaspases in some other species have demonstrated that metacaspases are autoproteolytically processed (Meslin et al., 2007; Wen et al., 2013). To confirm whether $\mathrm{TgMCA}$ undergoes proteolytic processing, endogenous TgMCA was fused with HA-tag through plic-HA vector knocking in at the C-terminal of the TgMCA locus in T. gondii (Figure 2D). The lysate of the tachyzoites of which TgMCA was marked with HA reacted with anti-HA monoclonal antibody using western blot. The results showed that an $\sim 80 \mathrm{kDa}$ band and a $70 \mathrm{kDa}$ band reacted against the antigen (Figure $2 \mathbf{B}$ ), and $\mathrm{TgAct}$ was used as control (Figure 2C). Taken together, these results suggest that TgMCA could be autoprocessed in tachyzoites.

Indirect immunofluorescence assay (IFA) analyzed the subcellular localization of $\mathrm{TgMCA}$ in $\mathrm{RH} \Delta \mathrm{ku} 80$ using mouse anti-rTgMCA serum (Figure 2E). TgMCA localized in the cytoplasm of the intracellular parasites. However, interestingly, the $\mathrm{TgMCA}$ of the extracellular parasites translocated into the nucleus and was concentrated in the nucleus of the parasites, co-localized with the nucleus DNA dye Hoechst. This results suggest execution of function of TgMCA is in the nucleus of the extracellular parasites.

\section{TgMCA Knockout, Complementary, and Overexpression Strains were Successfully Constructed}

To characterize the biological role of $\mathrm{TgMCA}$, we generated a complete knockout mutant of $T g$ MCA $(\triangle T g M C A)$ by targeting the native $T g M C A$ locus in the $\mathrm{RH} \Delta \mathrm{ku} 80$ strain, using a homologous recombinant approach to replace the TgMCA gene with a CAT-RFP cassette in the parasite genome (Figures 3A,B). Successfully targeted gene deletion was confirmed by PCR and western blot (Figures 3C,D, Table S4), and the parasites were resistance to chloramphenicol and the RFP was well distributed in the cytoplasm of the parasites. Western blot analysis showed no reactivity of $\triangle T g \mathrm{MCA}$ using antibodies against $\mathrm{TgMCA}$, with antibodies against $\mathrm{TgActin}$ as control. Re-expression of TgMCA was accomplished by transfection into $\triangle T g M C A$ with the Crispr/Cas9-UPRT vector and complete TgMCA coding sequence bearing the HA-tag at the C-terminal under the GRA1 promoter (Figure 3E). The expression of TgMCA was at high level in the complementary strain by western blot as the strong promoter (Figure 3F) and named $\triangle T g M C A-$ $\mathrm{cm}$. We also constructed a TgMCA overexpression strain (TgMCA OE), electroporated with modified pDMG-TgMCA into RH $\Delta$ ku80 (Figure S1A). After 10 generations of selection by pyrimethamine, a trangenic RH $\Delta$ ku8 stably expressing TgMCA 

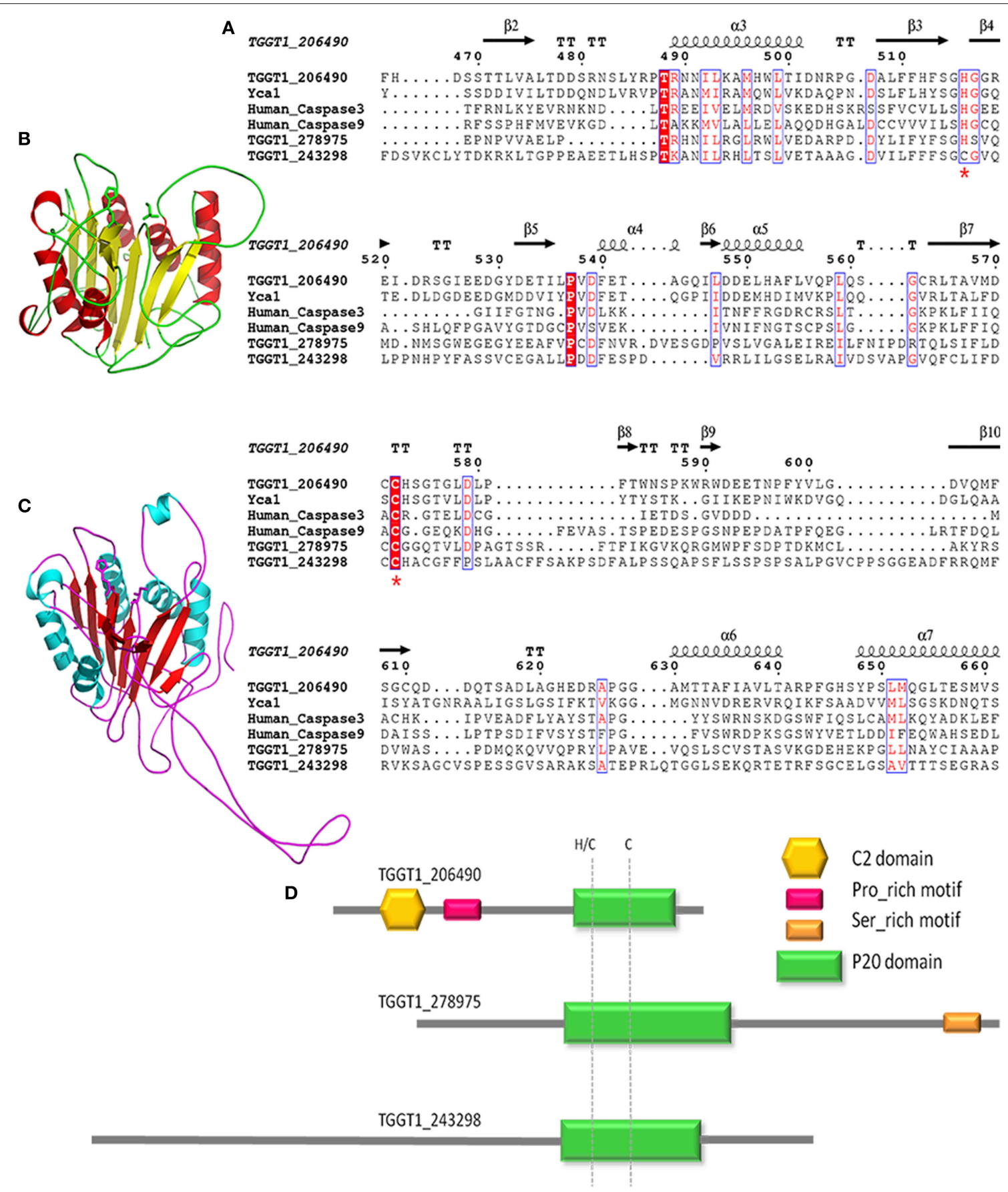

FIGURE 1 | Sequences-structure alignment of metacaspases. (A) TGGT1_206490, TGGT1_278975, and TGGT1_243298 protein sequence were aligned with sequences of human Caspase3, Caspase9, and yeast Yca1. The positions of identical and conserved residues in p20 domain are indicated by red-filled and non-filled rectangular frames, respectively. The conserved histidine and cysteine are indicated with red asterisk. Dots indicate gaps or missing residues. (B) The ternary structure of TgMCA was predicted by a model based on Yca1 (C) according to SWISS-Model and exhibited by Pymol. (D) Model pattern of the three ICE family proteins containing p20 domains in T. gondii, from top to bottom of the pattern, in order, TGGT1_206490, TGGT1_278975, and TGGT1_243298.

fused with the HA-tag was isolated by limiting dilution in 96well plates and confirmed by western blot (Figure S1B). To ensure the role of $\mathrm{TgMCA}$ in the overexpression strain was not affected by the plasmid pDMG-TgMCA insertion in the genome of the parasites, two clones were selected for further investigation.

\section{TgMCA is Required for Apoptosis Response of Tachyzoites}

In our previous study, we observed that tachyzoites isolated from a host cell exposed to the extracellular environment would undergo apoptosis by the TUNEL assay, whereas intracellular 

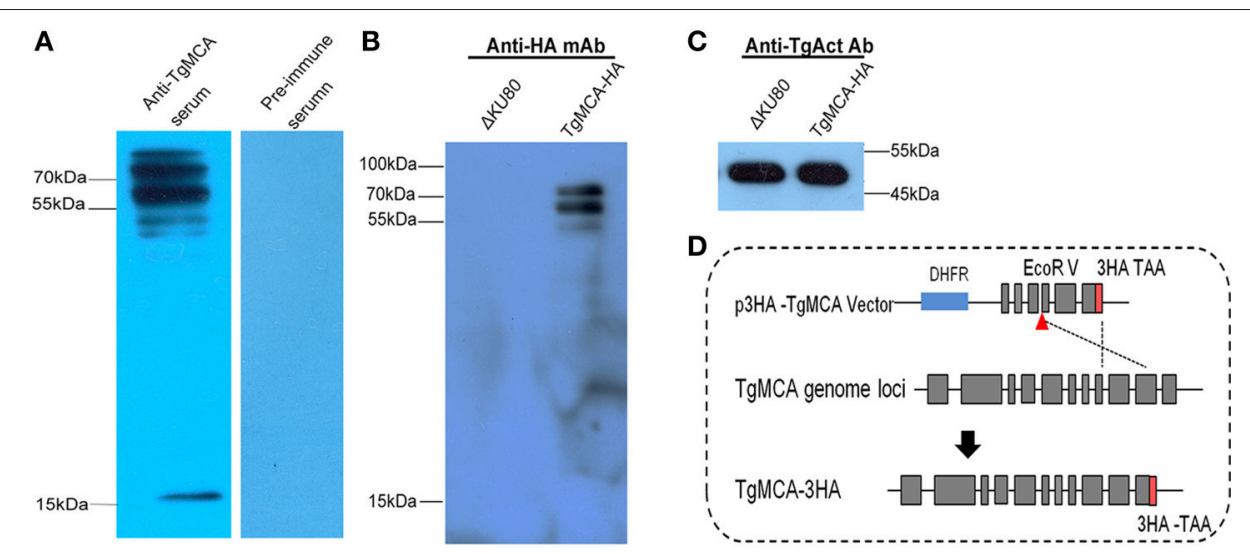

E
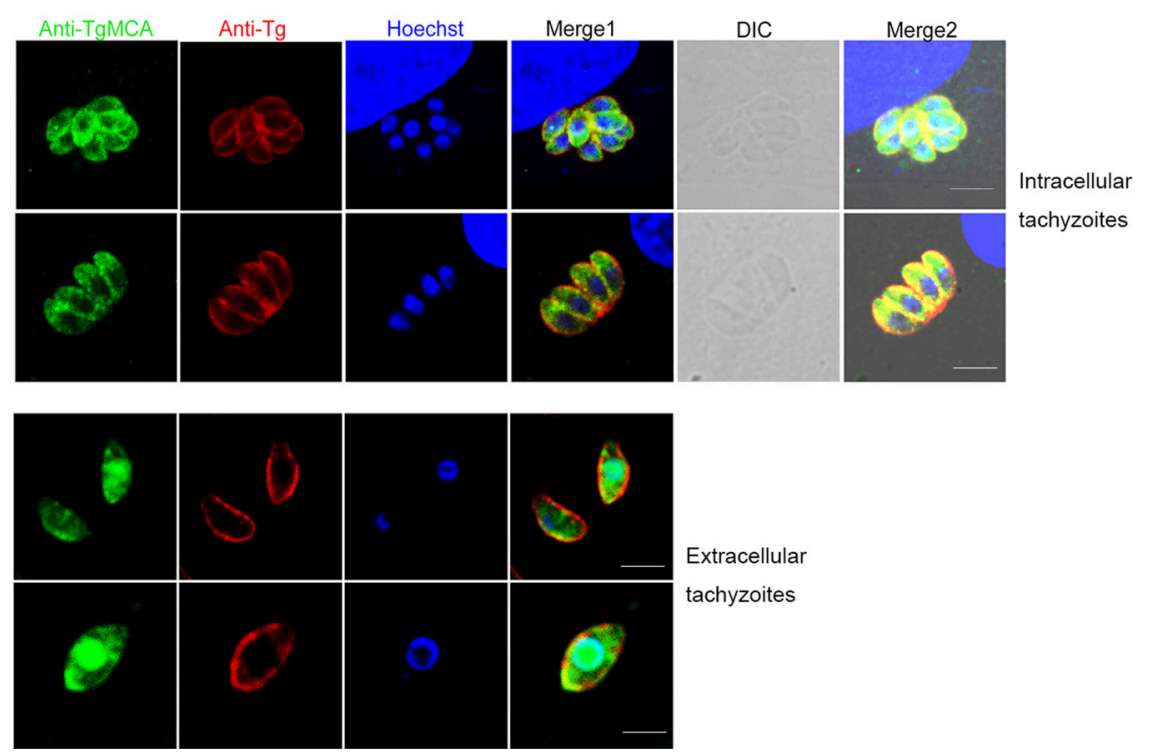

FIGURE 2 | Identification of TgMCA and cellular localization. (A) Western blot analysis of native TgMCA. Total antigens from cell cultured RH $\triangle$ ku80 tachyzoites. An expected band and two other bands were elicited by anti-rTgMCA polyclonal antibody. Pre-immune serum was used as control. (B) Total antigens from transgenic tachyzoites with TgMCA-HA and $\mathrm{RH} \Delta$ ku80, two expected bands were revealed using anti-HA monoclonal antibody as primary antibody. (C) TgActin used as control for (B). (D) Schematic of experimental design of TgMCA endogenously marked HA. A knock-in vector was constructed to target TgMCA endogenously marked HA at its C-terminal. (E) IFA analysis of TgMCA localization. TgMCA was distributed in the cytoplasm of the intracellular parasites and relocated to the nucleus of tachyzoites in the extracellular medium. The tachyzoites were stained with mouse anti-rTgMCA serum (green) or rabbit anti-T. gondii serum (red, stain the shape of the parasites), and the nucleus DNA was stained with Hoechst (blue). Scale bar, $5 \mu \mathrm{m}$.

parasites sheltered in the PVs would proliferate well and not become apoptotic without drug treatment (Nyoman and Lüder, 2013). To study the potential role of TgMCA in the apoptosis pathway of the parasites, we used the TUNEL assay to quantify the apoptotic ratios of $\Delta \mathrm{ku} 80, \Delta T g \mathrm{MCA}, \Delta T g \mathrm{MCA}-\mathrm{cm}$, and TgMCA OE strains treated with extracellular buffer for $4 \mathrm{~h}$. We found that the number of TUNEL-positive $\triangle T g M C A$ parasites was $17.93 \%$ significantly lower than that for $\Delta \mathrm{ku} 80$, which is $33.83 \%$ after exposure to extracellular buffer (Figure 4A), and apoptosis of $\Delta T g \mathrm{MCA}-\mathrm{cm} 1$ and $\Delta T g \mathrm{MCA}-\mathrm{cm} 2$ parasites were increased with the high level expression of $\mathrm{TgMCA}$ under GRA1 promoter (Figure 4A). The TgMCA OE strains were sensitive to the extracellular environment, with TUNEL-positive parasites at 53.8 and $49.35 \%$ (Figure $4 \mathbf{A}$ ). We also counted the number of apoptotic-response parasites in extracellular buffer during $1-6 \mathrm{~h}$. Figure $4 \mathrm{~B}$ shows that $\sim 5 \%$ of both parasites were TUNEL positive $(4.82 \%$ for $\triangle \mathrm{ku} 80$ and $4.73 \%$ for $\triangle \mathrm{TgMCA})$ for $1 \mathrm{~h}$. Then the apoptotic ratio of the parasites was evaluated by ionic stimulation, but $\triangle T g M C A$ still showed an obviously lower number of TUNEL-positive parasites, which indicates that $\mathrm{TgMCA}$ participates in the apoptotic-response pathway. Meanwhile, the mean fluorescence intensity of the images were obtained by confocal microscopy and analyzed. The FITCfluorescence intensity (TUNEL positive) of $\triangle \mathrm{TgMCA}$ was lower than for $\Delta \mathrm{ku} 80$ (Figure S2A), which corroborated the results above.

The transcription level of the other two ICE family proteins were detected by real-time PCR. According to three independent 


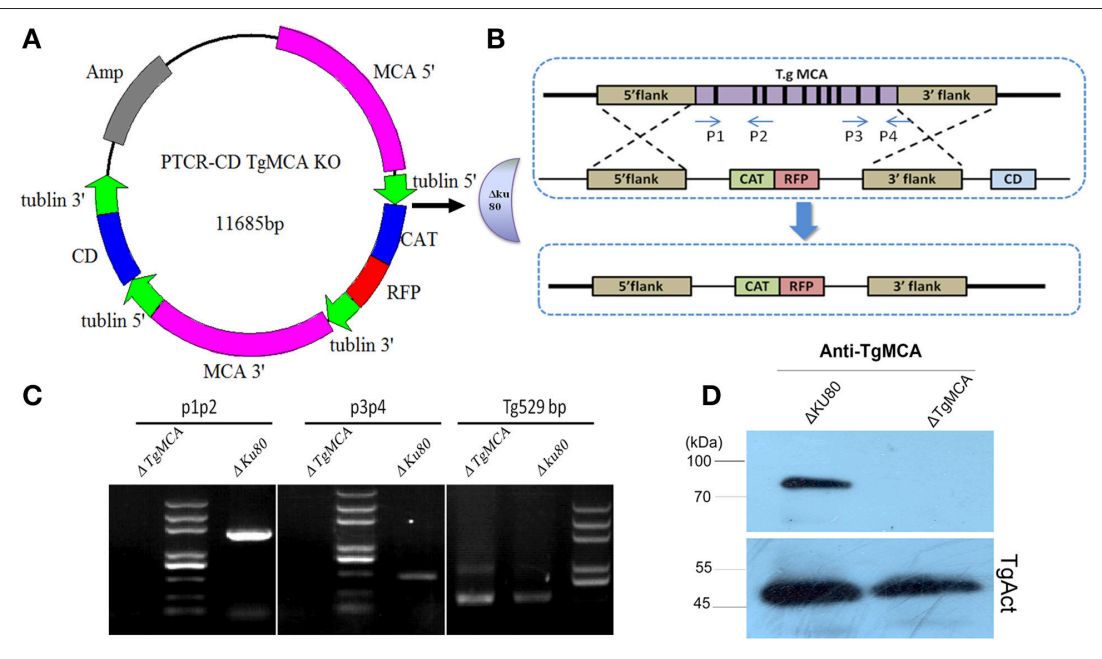

E
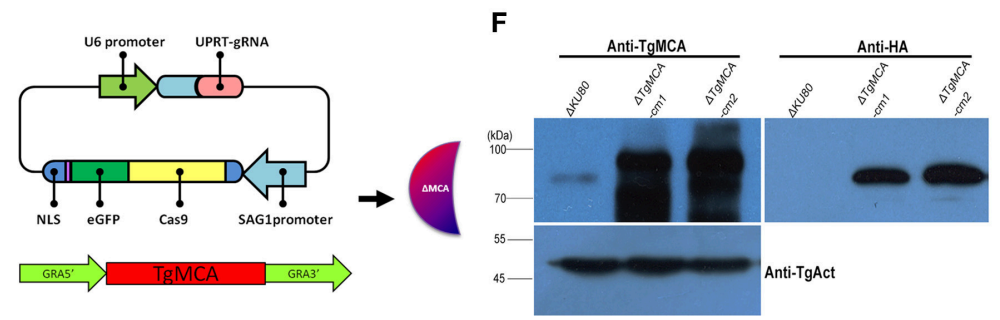

FIGURE 3 | Generation of a TgMCA mutant strain. (A,B) Schematic of the experimental design of the TgMCA knockout strain. A knockout vector (PTCR-CD TgMCA KO) was constructed to target the TgMCA complete gene. (C) Genomic PCR analysis of $\triangle T g M C A$ strain. The position of the primers are shown in (B). P1, P2 and P3, P4 were used to amplify exon2 to exon3 and exon10 to exon11 of TgMCA, respectively. (D) Western blot performed with anti-rTgMCA antibody on total extracts from $\triangle \mathrm{ku} 80$ and $\triangle T g M C A$, with TgActin used as control. (E) Schematic of the complementary strain construction. Crispr/Cas9-UPRT was used to target the UPRT locus of $\triangle T g M C A$, and TgMCA coding sequence fused with the HA-tag at the C-terminal under the GRA1 promoter was inserted into the gap of the UPRT locus. (F) Western blot analysis of the restoration of TgMCA with anti-rTgMCA serum and anti-HA antibody on total lysate of $\triangle \mathrm{ku} 80$ and $\triangle T g M C A-c m$, with $T g A c t i n$ used as control.

real-time PCR experiment, no significant transcriptional difference in TGGT1_278975 and TGGT1_243298 occurred in the extracellular $\triangle T g M C A$ compared to $\Delta \mathrm{ku} 80$ (Figure S2B, primers in Table S5).

\section{TgMCA Knockout Didn't Influence the Growth of Parasites In vitro or Virulence In vivo}

Because our results showed that TgMCA was involved in the pathway of apoptosis of $T$. gondii, we therefore investigated the invasion, replication, and virulence of $\triangle \mathrm{TgMCA}$ and $\mathrm{TgMCA}$ OE. The invasion ability of $\triangle \mathrm{TgMCA}, \Delta \mathrm{ku} 80$, and TgMCA $\mathrm{OE}$, all freshly isolated from Vero cells, were almost the same; however, after treatment with extracellular buffer for $1 \mathrm{~h}$, $\triangle T g M C A$ exhibited a stronger capacity of invasion than $\Delta \mathrm{ku} 80$ and TgMCA OE (Figure 5D). The lack of TgMCA did not affect parasite growth in vitro, as the plaque area of $\Delta T g \mathrm{MCA}$ were equivalent to $\Delta \mathrm{ku} 80$ (Figures $5 \mathbf{A}, \mathbf{B}$ ). The number of tachyzoites in each PVs of $\triangle T g M C A$ and $\Delta$ ku80 were almost the same, which indicated TgMCA knockout did not affect in the replication of the parasites. More interesting, overexpression of TgMCA affected proliferation of the parasites, as the plaque area of $\mathrm{TgMCA} \mathrm{OE}$ was smaller than the other two strains and $16.5 \%$ PVs contained only 1 tachyzoites according to the replication assay (Figures $\mathbf{5 A - C}$ ). The results above suggest that TgMCA knockout strain show more viability after exposed to the extracellular buffer but did not affect the parasite growth and replication, and overexpression of $\mathrm{TgMCA}$ affected the proliferation of the parasites in vitro.

Finally, we evaluated the contribution of $\mathrm{TgMCA}$ to the virulence of parasites in vivo. We infected mice intraperitoneally with 10 tachyzoites or 100 tachyzoites of $\Delta T g \mathrm{MCA}, \Delta \mathrm{ku} 80$, and TgMCA OE. We observed there was no significant difference (only 1 day) in survival time between mouse in the group infected with 100 tachyzoites of the different strains (Figure 5E). But mice infected with 10 tachyzoites of $\triangle \mathrm{ku} 80$ and TgMCA OE showed a significantly delayed time (7-19 days) to death compared to $\triangle T g M C A$ (Figure $5 \mathbf{F}$ ), indicating that $\triangle T g M C A$ was more virulent which correlated to the apoptotic function of TgMCA. Furthermore, 25\% mouse infected with 10 tachyzoites of TgMCA OE were resistant to the acute inflammatory period and survival. The results above suggest that $\mathrm{TgMCA}$ is not involved in parasite growth and replication or function of $\mathrm{TgMCA}$ is partially redundant, though a significantly delayed time to death among $\Delta$ TgMCA, $\Delta \mathrm{ku} 80$, and TgMCA OE at the dose of 10 parasites. 


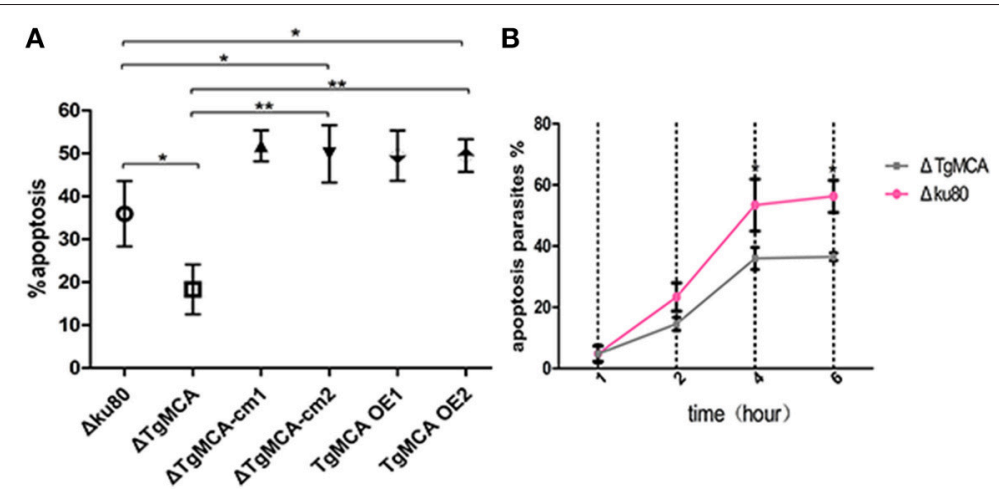

FIGURE 4 | Extracellular $\triangle T$ TMCA is less sensitive to environmental stress. (A) Ratio of apoptotic parasites by TUNEL assay. Apoptotic $\triangle T g M C A$ was significantly decreased when treated with BAG buffer for $4 \mathrm{~h}(p \leq 0.05)$. TgMCA OE $\triangle$ TgMCA-cm 1 and $\triangle$ TgMCA-cm2 produced far more TUNEL-positive parasites than $\Delta$ ku80 $(p \leq 0.05, p \leq 0.01)$. Asterisks indicate statistically significant results as determined by One-way ANOVA with Tukey's post-hoc comparison. (B) Ratio of apoptotic parasites pre-incubated in Ringer buffer for $1-6 \mathrm{~h}$ by TUNEL assay. Both strains showed increased apoptotic ratio. Apoptotic $\Delta$ ku80 was higher than $\triangle T g M C A$ after $2 \mathrm{~h}$ in extracellular buffer and significantly increased at $4 \mathrm{~h}(p \leq 0.05)$. Asterisks indicate statistically significant results $(p \leq 0.05)$ as determined by Two-way ANOVA with Tukey's post-hoc comparison. Data are mean \pm SD (error bars) of three independent experiment.

\section{DISCUSSION}

The phenotype of the $\operatorname{TgMCA}$ gene complete knockout strain was analyzed in our study. Metacaspases exist in many nonmetazoan species, sharing common structural features with caspase. They are able to use the conserved catalytic activity of the cysteine and histidine dyad as a nucleophile to hydrolyze the substrate peptide downstream (McLuskey et al., 2012). A pro-cell death role of metacaspases has been identified in fungi, protozoa, and plants (Meslin et al., 2011; Tsiatsiani et al., 2011). Nyoman et al. showed that caspase-like protease activity was clearly required during miltefosine-induced apoptosis in T. gondii, and the caspase inhibitors E64 and Z-VAD-fmk completely abolished drug-induced TUNEL-positive parasites, indicating that caspaselike activity is essential in the apoptotic pathway of $T$. gondii (Nyoman and Lüder, 2013). We have found several caspaselike proteases by a bioinformatics search in ToxoDB, including three ICE family members containing p20 domains and some other cysteine proteases. We found that TGGT1_206490 was the nearest to yeast Yca1, compared to TGGT1_278975 and TGGT_243298. Previous in vitro studies of Ycal have shown that the catalytic residues Cys and His are necessary for hydrolysis (Wong et al., 2012). Therefore, we hypothesize TGGT1_206490 maintains the hydrolysis ability because of the conserved Cys-His catalytic dyad.

We obtained a strain of $T$. gondii tachyzoites lacking the TgMCA gene by gene knockout. First, we analyzed the apoptotic cell death of $\triangle T g M C A$ and the parental strains. For apoptosis analysis, $\Delta T g \mathrm{MCA}$ and $\Delta \mathrm{ku} 80$ freshly isolated from the host cell were pre-incubated in extracellular buffer at $37^{\circ} \mathrm{C}$ for different time. We found that both strains, when freshly isolated (washed the cultures with PBS 3 times and removed the extracellular parasites 3 and harvested the freshly egressed parasites within $30 \mathrm{~min}$ ), showed no TUNEL-positive signal, and $\triangle \mathrm{TgMCA}$ and $\Delta \mathrm{ku} 80$ showed similar ratio of apoptosis when they were exposed to extracellular buffer for $1 \mathrm{~h}$. Though there was no remarkable difference in the TUNEL labeling assay for $1 \mathrm{~h}, \Delta \mathrm{TgMCA}$ showed greater viability than $\Delta \mathrm{ku} 80$ by the method of invasion statistics. Over time, both strains gradually underwent apoptosis during 2$6 \mathrm{~h}$, but the apoptotic ratio of $\triangle T g M C A$ remained significantly lower than that of $\Delta \mathrm{ku} 80$. In our research, the ratio of apoptotic tachyzoites was different from previous reports (Nyoman and Lüder, 2013) due to different methods of stimulation. In addition, both the TgMCA OE and $\triangle T g M C A-c m$ strains were sensitive to the extracellular buffer, which also confirmed the apoptotic function of $\mathrm{TgMCA}$, as the same in some other organisms of which metacaspases over-expressed (Lee et al., 2007; Coll et al., 2010). Based on the process of apoptosis in $\triangle T g M C A$ and $\Delta \mathrm{ku} 80$, we believe that TgMCA plays a role in regulating tachyzoite apoptosis. Nevertheless, a small quantity of $\triangle T g M C A$ were still TUNEL positive, and it is unclear whether other PCD-related proteases might be responsible for the apoptosis of $T$. gondii. Some characterizations of the early stage of apoptosis, such as externalization of phosphatidylserine and loss of mitochondrial membrane potential, have been previously reported, which were not performed in our study. Since caspases are involved in signaling pathways downstream of apoptosis (Carwford and Wells, 2011), the TUNEL assay was an effective and necessary approach in this study. But according to the result of TUNEL assay, the mechanism of the apoptosis of T. gondii is still unclear. The alternative experiments such as mitochondrial membrane potential test and the distribution of cytochrome $\mathrm{C}$ should be involved to reveal the apoptosis pathway of T. gondii, so that the specific TgMCA role would be more strengthened.

The ionic concentration of extracellular buffer was almost the same as the ionic concentration of extracellular fluid. Extracellular T. gondii both in vivo and in vitro undergoes apoptosis (Santos et al., 2011; Nyoman and Lüder, 2013), which indicated that egress tachyzoites which are unable to invade another host cell will undergo active death. DNA fragmentation is among the downstream hallmarks of apoptosis in mammals as well as protozoa (Jiménez-Ruiz et al., 2010; Proto et al., 

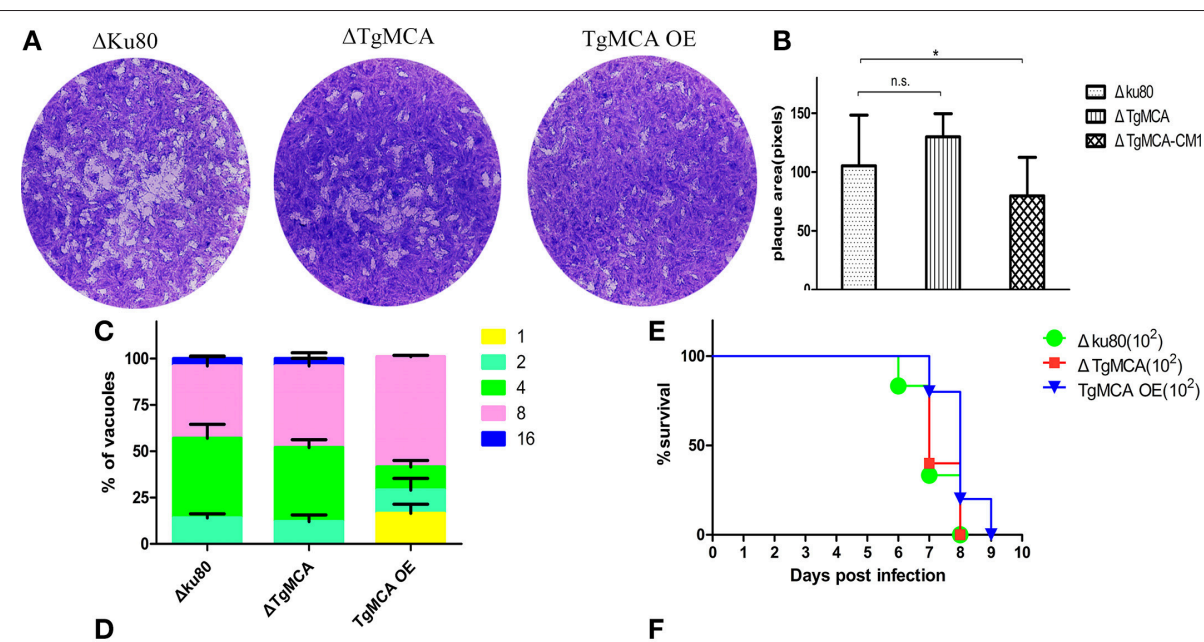

D

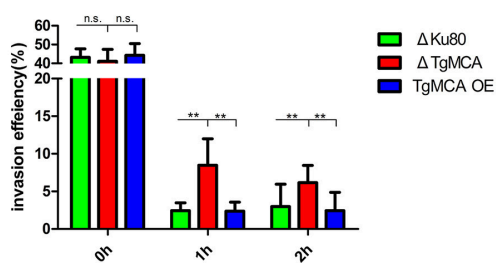

$\mathbf{F}$

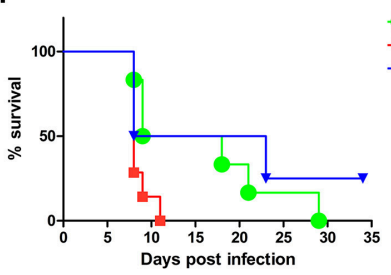

FIGURE 5 | Lack ofTgMCA did not affect intracellular parasite replication but did affect parasite viability in the extracellular environment. (A) Plaque assay comparing growth of $\triangle \mathrm{TgMCA}, \triangle \mathrm{ku} 80$, and TgMCA OE. Each well (HFF cell) was infected with 500 parasites, and plaques were stained 7 days later. Data were compiled from three independent assay. (B) The plaque areas were counting by randomly chosen at least 50 plaques and using the Pixel point in Photoshop C6S software (Adobe, USA), and the data were compiled from three independent experiment. Analysis of plaque area was performed using One-way ANOVA with Tukey's post-hoc comparison. Asterisks indicated significant results $(p \leq 0.05)$. (C) Intracellular parasite replication of $\triangle T g M C A$, $\triangle \mathrm{ku} 80$, and TgMCA OE. Data were compiled from three independent assay, and in each assay 100 total PVs of each strain were counted. Data were determined by Chi-square analysis. (D) Invasion ratio of $\triangle T$ TMCA, $\triangle$ ku80, and TgMCA OE, freshly isolated and pre-incubated in extracellular buffer for 1 or $2 \mathrm{~h}$. The ratio was based on the number of cells infected with parasites divided by the number of total cells in one field of view. Asterisks indicated statistically significant results $(p \leq 0.05$ as determined by Two-way ANOVA with Tukey's post-hoc comparison). Data are mean \pm SD (error bars) of three independent experiment. (E,F) Mouse survival after infection with different doses of $\Delta$ TgMCA, $\Delta \mathrm{ku} 80$, and TgMCA OE. Balb/c mouse were injected intraperitoneally (i.p.) with 100 or 10 indicated parasites. There were 5 female mice in each group, statistical analysis was performed using life test (life test data = surv) in statistical analysis system (SAS institute Inc., USA). The figures are representative of three experiments (a dose of 100 tachyzoites) and two experiments (a dose of 10 tachyzoites) with similar outcomes.

2013). The cysteine proteases, caspases or metacaspases, are involved in the apoptosis pathway, which may trigger DNA fragmentation directly and indirectly. It is very interesting to observe the different localization of $\mathrm{TgMCA}$ in intracellular and extracellular parasites, and the change of localization may reveals to the function of $\mathrm{TgMCA}$, which indicates that it is possible to cleave substrate and trigger DNA fragmentation, ultimately leading to parasite death. However, it is regrettable that we failed to find the downstream substrate peptide that TgMCA target to hydrolyze, as the interaction may have occurred instantaneously. Some studies have indicated that the conserved protein Tudor staphylococcal nuclease (TSN), which participates in activating transcription, is the natural substrate of metacaspase (Sundström et al., 2009). Although TSN exists in Toxoplasma, whether it is the target protein of metacaspase and essential for the apoptosis of Toxoplasma are an important issue for further study.

Based on the phenotypic assay, $\triangle T g M C A$ showed no obvious difference in growth in HFF and Vero cells or virulence in vivo compared to $\Delta \mathrm{ku} 80$. Therefore, TgMCA is not an obligatory factor for T. gondii invasion and proliferation, or redundant proteases are involved. Though TgMCA OE strain showed slower growth in vitro, the mechanism hasn't been understood. Up to now, we have generated TGGT1_278975 knockout strain and double genes (TGGT1_278975 and TGGT1_206490) knockout strain (data not shown). We observed that the lack of TGGT1_278975 did not affect the apoptosis of the parasites, but lack of both TGGT1_278975 and TGGT1_206490 has an effect on the proliferation of the parasites significantly (data not shown). We hypothesize the ICE family protein are involved in proliferation of $T$. gondii, but the mechanism has not been clear. Metacaspase studies of other organisms such as yeast (Lee et al., 2010; Hill et al., 2014) and T. brucei (Helms et al., 2006) showed multiple functions in protein aggregation and cell proliferation. Whether TgMCA may be responsible for non-cell death functions should be explored in the future. The function of the other two protein have been our focused research recently.

As we all known, virulence assay of mouse infected with dose of 10 tachyzoites are easier to misinterpret as the smaller amount the greater error, and the time taking to count would 
influence the viability of the parasites. However, we believe that the significant difference in survival time when mouse infected with 10 tachyzoites is due to the much more viability of $\Delta T g \mathrm{MCA}$ compared to $\triangle \mathrm{ku} 80$ and $T g \mathrm{MCA}$ OE. The increased invasion number of $\triangle T g M C A$ compared to $\Delta \mathrm{ku} 80$ when both were preincubated in extracellular buffer was also correlated with the apoptotic role of $\mathrm{TgMCA}$ in $T$. gondii, as the more viable parasites are, the higher invasion ratio is.

Apoptosis is known in the context of PCD in multicellular individuals, which contributes to the functionality of the organism. Such active cell death has also been observed in unicellular organisms, which seems contrary to evolutionary theory. Despite a lack of evidence regarding the molecular mechanisms of the apoptosis pathway, the benefits of apoptosis as an altruistic behavior have been proposed over the past several years. First, it can control the parasite population to maintain optimal conditions in a host to enable propagation (Bruchhaus et al., 2007). Second, it regulates inflammation of a host by apoptotic parasites induced anti-inflammatory secretions, which seem to be essential for the development of the rest of the population. Van Zandbergen et al. reported that apoptotic Leishmania promastigotes could induce TGF- $\beta$ secretion, thereby enabling the intracellular survival of viable parasites in an altruistic way (van Zandbergen et al., 2006). Another research demonstrated that externalization of phosphatidylserine was necessary for successful Toxoplasma infection, thus indicating that PS+ and PS - subpopulations are required to maintain the balance between inflammation and parasite growth (Santos et al., 2011).

In summary, the reduced apoptotic cell death and greater viability of $\triangle T g \mathrm{MCA}$ indicated that $\mathrm{TgMCA}$ performs an important function in $\mathrm{T}$. gondii apoptosis. $\mathrm{TgMCA}$ was not observed to play a role in propagation from the current result,

\section{REFERENCES}

Bruchhaus, I., Roeder, T., Rennenberg, A., and Heussler, V. T. (2007). Protozoa parasites: programmed cell death as mechanism of parasitism. Trends Parasitol. 23, 376-383. doi: 10.1016/j.pt.2007.06.004

Carmona-Gutierrez, D., Fröhlich, K. U., Kroemer, G., and Madeo, F. (2010). Metacaspases are caspase. Doubt no more. Cell Death Differ. 17, 377-378. doi: 10.1038/cdd.2009.198

Carwford, E. D., and Wells, J. A. (2011). Caspase substrates and cellular remodeling. Annu Rev. Biochem. 80, 1055-1087. doi: 10.1146/annurevbiochem-061809-121639

Choi, C. J., and Berges, J. A. (2013). New types of metacaspases in phytoplankton reveal diverse origins of cell death proteases. Cell Death Dis. 4:e490. doi: 10.1038/cddis.2013.21

Coll, N. S., Varcammen, D., Smidler, A., Clover, C., Van Breusegem, F., and Dangl, J. L. (2010). Arabidopsis type I metacaspases control cell death. Science 330, 1393-1397. doi: 10.1126/science.1194980

Dubey, J. P. (2010). Toxoplasmosis of Animals and Human, 2nd Edn. Boca Raton, FL: CRC press.

Elomre, S. (2007). Apoptosis: a review of programmed cell death. Toxicol. Pathol. 35, 495-516. doi: 10.1080/01926230701320337

Gannavaram, S., and Debranbant, A. (2012). Programmed cell death in Leshimania: biochemical evidence and role in parasite infectivity. Front. Cell. Infect. Microbiol. 2:95. doi: 10.3389/fcimb.2012. 00095 although multifunction of metacaspases have been confirmed in other species. Our studies demonstrated that TgMCA plays apoptotic role in T. gondii.

\section{AUTHOR CONTRIBUTIONS}

QL, JL, and ML conceived and designed the experiments. LM and ML performed the experiments. QL, PH, and ML analyzed the data. QL and JL contributed reagents, materials and analysis tools. QL and ML wrote the paper.

\section{FUNDING}

This study was supported by National Natural Science Foundation of China (No. 31372424) and the National Key Basic Research Program (973 program) of China (No. 2015CB 150300).

\section{ACKNOWLEDGMENTS}

We sincerely thank Professor Silvia Moreno (University of Georgia, USA) for kindly providing the PTCY vector and $\mathrm{RH} \Delta \mathrm{ku} 80$. We are grateful to Professor BangShen (Huazhong Agricultural University) to kindly providing CRISPR/CAS9UPRT vector. We are sincerely thank Professor Xuenan Xuan (Obihrio University of Agriculture and Veterinary Medicine, Japan) to kindly providing PDMG vector.

\section{SUPPLEMENTARY MATERIAL}

The Supplementary Material for this article can be found online at: http://journal.frontiersin.org/article/10.3389/fmicb. 2015.01560
Helms, M. J., Ambit, A., Appleton, P., Tetley, L., Coombs, G. H., and Mottram, J. C. (2006). Bloodstream form Trypanosoma brucei depend upon multiple metacaspases associated with RAB11-positive endosomes. J. Cell Sci. 119, 1105-1117. doi: 10.1242/jcs.02809

Hill, S. M., Hao, X. X., Liu, B. D., and Nyström, T. (2014). Life-span extension by a metacaspase in the yeast Saccharomyces cerevisiae. Science 344, 1389-1392. doi: $10.1126 /$ science. 1252634

Jiménez-Ruiz, A., Alzate, J. F., Macleod, E. T., Lüder, C. G. K., Fasel, N., and Hurd, H. (2010). Apoptotic markers in protozoan parasites. Parasit. Vectors 3:104. doi: 10.1186/1756-3305-3-104

Kaczanowski, S., Sajid, M., and Reece, S. E. (2011). Evolution of apoptosis-like programmed cell death in unicellular protozoan parasites. Parasit. Vectors 4:44. doi: 10.1186/1756-3305-4-44

Kosec, G., Alvarez, V. E., Agüero, F., Sánchez, D., Dolinar, M., and Turk, B. (2006). Metacaspases of Trypanosoma cruzi: possible candidates for programmed cell death mediators. Mol. Biochem. Parasitol. 145, 18-28. doi: 10.1016/j.molbiopara.2005.09.001

Laverrière, M., Cazzulo, J. J., and Alvarez, V. E. (2012). Antagonic activities of Trypanosoma cruzi metacaspase affect the balance between cell proliferation, death and differentiation. Cell Death Differ. 19, 1358-1369. doi: $10.1038 /$ cdd. 2012.12

Lee, N., Gannavaram, S., Selvapandiyan, A., and Debrabant, A. (2007). Characterization of metacaspases with trypin-like activity and their putative role in programmed cell death in protozoa parasites Leishmania. Eukaryot. Cell 6, 1745-1757. doi: 10.1128/EC.00123-07 
Lee, R. E., Brunette, S., Puente, L. G., and Megeney, L. A. (2010). Metacaspase Ycal is required for clearance of insoluble protein aggregates. Proc. Natl. Acad. Sci. U.S.A. 107, 13348-13353. doi: 10.1073/pnas.1006610107

Madeo, F., Herker, E., Maldener, C., Wissing, S., Lächelt, S., Herlan, M., et al. (2002). A caspase-related protease regulates apoptosis in Yeast. Mol. Cell 9, 911-917. doi: 10.1016/S1097-2765(02)00501-4

McLuskey, K., Rudolf, J., Proto, W. R., Isaacs, N. W., Coombs, G. H., Moss, C. X., et al. (2012). Crystal structure of a Trypanosoma brucei metacaspase. Proc. Natl. Acad. Sci. U.S.A. 109, 7469-7474. doi: 10.1073/pnas.1200885109

Meslin, B., Barnadas, C., Boni, V., Latour, C., Monbrison, F., Kaiser, K., et al. (2007). Features of apoptosis in Plasmodium falciparum Erythrocytic stage through a putative role of PfMCA1 metacaspase-like protein. J. Infect. Dis. 195, 1852-1859. doi: 10.1086/518253

Meslin, B., Zalila, H., Fasel, N., Picot, S., and Bienvenu, A. L. (2011). Are protozoan metacaspases potential parasite killers? Parasit. Vectors 4:26. doi: 10.1186/17563305-4-26

Nyoman, A. D., and Lüder, C. G. K. (2013). Apoptosis-like cell death pathways in the unicellular parasite Toxoplasma gondii following treatment with apoptosis inducers and chemotherapeutic agents: a proof-of-concept study. Apoptosis 18, 664-680. doi: 10.1007/s10495-013-0832-8

Proto, W. R., Coombs, G. H., and Mottram, J. C. (2013). Cell death in parasitic protozoa: regulated or incidental? Microbiology 11, 58-66. doi: 10.1038/nrmicro2929

Reece, S. E., Pollitt, L. C., Colegrave, N., and Gardner, A. (2011). The meaning of death: evolution and ecology of apoptosis in protozoan parasites. PLoS Pathog. 7:e1002320. doi: 10.1371/journal.ppat.1002320

Sambrook, J., and Russell, D. (2000). Molecular Cloning. New York, NY: Cold Spring Harbour Laborartory Press.

Santos, T. A., Portes, J. A., Damasceno-Sá, J. C., Caldas, L. A., Souza, W., DaMatta, R. A., et al. (2011). Phosphatidylserine exposure by Toxoplasma gondii is fundamental to balance the immune response granting survival of the parasite and of the host. PLoS ONE 6:e27867. doi: 10.1371/journal.pone. 0027867

Shen, B., Brown, K. M., Lee, T. D., and Sibley, L. D. (2014). Efficient gene disruption in diverse strains of Toxoplasma gondii using CRISP/CAS9. MBio 5:e01114. doi: 10.1128/mBio.01114-14

Suarez, M. F., Filonova, L. H., Smertenko, A., Savenkov, E. I., Clapham, D. H., and von Arnold, S. (2004). Metacaspase-dependent programmed cell death is essential for plant embryogenesis. Curr. Biol. 14, R339-R340. doi: 10.1016/j.cub.2004.04.019

Sundström, J. F., Vaculova, A., Smertenko, A. P., Savenkov, E. I., Golovko, A., and Minina, E. (2009). Tudor staphylococcal nuclease is an evolutionarily conserved component of the programmed cell death degradome. Nat. Cell Biol. 11, 347-1354. doi: 10.1038/ncb1979

Tsiatsiani, L., Breusegem, F. V., Gallois, P., Zavialov, A., Lam, E., and Bozhkov, P. V. (2011). Metacaspases. Cell Death Differ. 18, 1279-1288. doi: $10.1038 / \mathrm{cdd} .2011 .66$

Uren, A. G., O’Rourke, K., Aravind, L. A., Pisabarro, M. T., Seshagiri, S., Koonin, E. V., et al. (2000). Identification of paracaspases and metacaspases: two ancient families of caspase-like proteins, one of which plays a key role in MALT Lymphoma. Mol. Cell 6, 961-967. doi: 10.1016/s1097-2765(05)00086-9

van Zandbergen, G., Bollinger, A., Wenzel, A., Kamhawi, S., Voll, R., Klinger, M., et al. (2006). Leishmania disease development denponds on the presence of aopototic promastigotes in the virulent inoculum. Proc. Natl. Acad. Sci. U.S.A. 103, 13837-13842. doi: 10.1073/pnas.0600843103

Wanderley, J. L. M., Thorpe, P. E., Barcinski, M. A., and Soong, L. (2013). Phosphatidylserine exposure on the surface of Leishmania amazonesis amatigotes modulates in vivo infection and dendritic cell function. Parasite Immunol. 35, 109-119. doi: 10.1111/pim.12019

Wang, H., Lei, T., Liu, J., Li, M. Z., Nan, H. Z., and Liu, Q. (2014). A nuclear factor of high mobility group box protein in Toxoplasma gondii. PLoS ONE 9:e111993. doi: 10.1371/journal.pone.0111993

Wen, S., Ma, Q. M., Zhang, Y. L., Yang, J. P., Zhao, G. H., Fu, D. Q., et al. (2013). Biochemical evidence of key residues for the activation and autoprocessing of tomato type II metacaspase. FEBS Lett. 587, 2517-2522. doi: 10.1016/j.febslet. 2013.06.057

Wong, A. H. H., Yan, C. Y., and Shi, Y. G. (2012). Crystal structure of the yeast metacaspse Yca1. J. Biol. Chem. 287, 29251-29259. doi: 10.1074/jbc.M112. 381806

Zalila, H., González, I. J., EI-Fadili, A. K., Delgado, M. B., Desponds, C., Schaff, C., et al. (2011). Processing of metacaspase into a cytoplasmic catalytic domain mediating cell death in Leishmania major. Mol. Microbiol. 79, 222-239. doi: 10.1111/j.1365-2958.2010.07443.x

Conflict of Interest Statement: The authors declare that the research was conducted in the absence of any commercial or financial relationships that could be construed as a potential conflict of interest.

Copyright (c) $2016 \mathrm{Li}$, Wang, Liu, Hao, Ma and Liu. This is an open-access article distributed under the terms of the Creative Commons Attribution License (CC BY). The use, distribution or reproduction in other forums is permitted, provided the original author(s) or licensor are credited and that the original publication in this journal is cited, in accordance with accepted academic practice. No use, distribution or reproduction is permitted which does not comply with these terms. 